\title{
Strates
}

STRATES Matériaux pour la recherche en sciences sociales

$7 \mid 1993$

Témoins du monde : Bulgarie, identités chinoises, explorer l'île de France

\section{Autoportrait avec géographie}

Jacques Lévy

\section{(2) OpenEdition}

Journals

Édition électronique

URL : http://journals.openedition.org/strates/1171

DOI : $10.4000 /$ strates. 1171

ISSN : $1777-5442$

Éditeur

Laboratoire Ladyss

Édition imprimée

Date de publication : 30 juin 1993

ISSN : 0768-8067

Référence électronique

Jacques Lévy, " Autoportrait avec géographie », Strates [En ligne], 7 | 1993, mis en ligne le 20

décembre 2005, consulté le 08 septembre 2020. URL : http://journals.openedition.org/strates/1171 ;

DOI : https://doi.org/10.4000/strates. 1171

Ce document a été généré automatiquement le 8 septembre 2020

Tous droits réservés 


\title{
Autoportrait avec géographie
}

\author{
Jacques Lévy
}

\section{NOTE DE L'ÉDITEUR}

Cet article reprend des extraits de la troisième partie de la thèse de doctorat d'État L'espace légitime soutenue à l'Université de Paris 7 en janvier 1993, sous la direction d'Olivier Dollfus. L'essentiel de cette thèse sera prochainement publié.

«En somme, il faut se servir de tous les secours que peuvent fournir l'entendement,

l'imagination, les sens et la mémoire. » René Descartes, Règles pour la direction de

l'esprit, 1629.

... On peut affirmer sans trop de risque que la subjectivité des chercheurs fait partie des facteurs de production de la recherche. Comprendre comment cette subjectivité cognitive fonctionne devrait faire partie de l'entraînement à la recherche. En l'occurrence, il ne s'agit que d'un cas particulier et abordé en outre de manière spécifique, celle de la diachronie. J'ai voulu essayer de comprendre d'où venait une évolution, faite de continuités et de ruptures, qui avait été la mienne...

2 Il ne semble pas possible, en effet, de se passer des matériaux de sa propre histoire pour supporter et inventer le présent. Autant assumer cette réalité et inviter le lecteur à intégrer ce biais dans son analyse et dans son jugement...

3 Voici donc quelques séries d'informations, présentées indépendamment les unes des autres, afin d'aider le lecteur à se faire sa propre idée. Je ne prétends pas que ce sont des «matériaux bruts»-expression d'ailleurs méthodologiquement vide de sens. Il faut les prendre pour ce qu'ils sont, la réponse fragile et sans cesse reformulée à la question : «Quels sont les moments et les attitudes qui ont compté le plus dans ma vie intellectuelle?»

(...)

Cosmopolis 
5 Au-delà des voyages et des lectures, le monde - c'est-à-dire le monde extérieur, mais pris dans sa totalité - a été présent de diverses et fortes façons dans ma vie, dès le début. Il y a d'abord le cosmopolitisme des origines, attestées (Istanbul, Plovdiv et, plus exotique encore, Clermont-Ferrand) ou reconstruites (l'Espagne d'avant 1492...). Petitfils d'immigrés, je n'ai pourtant jamais eu, à ma connaissance, de problèmes d'identité comme on peut en rencontrer en pareil cas. C'est que, préparé par l'insertion économique et linguistique de leurs parents, mon père et ma mère avaient réalisé avant ma naissance un intense travail de production identitaire consistant à déplacer l'appartenance communautaire vers des entités à vocation universaliste: la nation française comme processus inachevé d'accomplissement de l'idéal de 1789-93 et le Parti communiste comme instrument d'une libération générale de l'homme.

6 Les communautés imposées, notamment celle ethnico-religieuse que leurs parents leur proposaient comme référence fondatrice, ou encore l'idéal patrimonial qui pouvait déboucher sur une hypostase métaphysique de la famille ou de la position économique, ils les avaient fermement rejetées dès leur adolescence. En outre, la guerre avait légitimé à chaud ces choix : mon père a été vraiment résistant. Je n'ai pas d'abord perçu la rareté relative de cet engagement, car lui-même, par modestie mais aussi en suivant l'esprit du temps, proposait une image que nous savons aujourd'hui enjolivée des attitudes de la société française de l'époque. Il n'empêche que cela a donné à l'éducation que j'ai reçue une grande sérénité humaniste dans le rapport aux autres " peuples » : pas de nationalisme, pas de laisser-aller à une xénophobie anti-allemande. Cette sécurité avait sans doute aussi ses défauts: elle engendrait une tranquillité morale et une assurance intellectuelle qui nous a conduits, eux et moi, vers une complaisance envers des causes tout aussi dangereuses.

7 Mais elle a eu au moins l'importance, je n'hésite pas à dire l'avantage, de m'éloigner de toute logique communautaire. Il est vrai que les juifs sépharades (= originaires de la péninsule ibérique), même demeurés hispanophones, ont développé un habitus communautaire faible, qui s'est atténué ou effacé au contact de sociétés qui valorisaient l'intégration. C'est le cas en France, où l'on voit assez nettement la différences avec les ashkénazes (= originaires d'Europe centrale et orientale), qui disposaient d'un appareil communautaire plus structuré et surtout avec les juifs «orientaux» (= originaires du monde arabe), parfois appelés à tort "sépharades ", et qui restent encore aujourd'hui porteurs d'attitudes communautaires caractéristiques des sociétés dont ils sont issus. Les antécédents étaient donc favorables, mais la rupture accomplie par mes parents n'en est pas moins essentielle, plus marquée que pour beaucoup de personnes de leur génération. Je n'ai fait de ce point de vue que prolonger leur démarche en refusant tout enrôlement dans une quelconque judéité, que certains éléments de mon entourage tentaient, sous couvert de définitions contradictoires, de me faire "avaler de force». J'ai sans hésitation répondu non, puisque tel était mon choix d'athée, de Français et de citoyen du monde, beaucoup plus attiré par le mythe de la Pentecôte que par celui du « peuple élu ». J'ai pu ensuite appliquer la méthode à d'autres communautés, affirmant quoi qu'il m'en coûte la supériorité du groupe choisi sur le groupe imposé, dans la famille, les colonies de vacances, les « collectifs de travail » ou les partis politiques.

8 J'ai ainsi été formé à un cosmopolitisme qu'on pourrait qualifier de troisième type : ni celui des colonisés ou des bannis, qui souffrent d'un pays perdu, ni celui des apatrides, qui souffrent d'un pays désiré. Un cosmopolitisme post-nationaliste, qui voit la nation comme un repère parmi d'autres, relatif. 
Ce monde n'était donc pas menaçant, mais empli de connaissances et de beautés, d'humanité. Il pouvait entrer dans la maison, par ses habitants reçus ou rencontrés, mais aussi par ses problèmes. Ce cosmos était une cité : l'on y faisait de la politique ou, si l'on en faisait pas, l'on aurait dû. La radio ou les journaux déversaient leur flot quotidien de surprises, immédiatement réinterprétées selon une grille d'analyse robuste : le monde n'allait pas toujours dans la direction souhaitée, mais on aurait pu dire à chaque fois ce qu'elle aurait pu être. Certaines nouvelles étaient bonnes et gratifiaient la journée d'une petite dose d'euphorie, d'autres, mauvaises, suscitaient l'indignation. Tout était intéressant, tout appelait l'action, et en prendre conscience était déjà agir. Je me suis senti, le matin en pyjama, en buvant mon chocolat avant de partir à l'école, comme un citoyen à part entière d'un monde intelligible. Je me souviens assez bien des événements de Suez en 1956 (j'avais quatre ans) : c'était inquiétant (l'essence risquait de manquer), c'était angoissant (la guerre était dans l'air), mais c'était aussi et d'abord un événement, une nouveauté formidable dont j'étais, moi comme les autres, partie prenante.

Le monde comme demeure, la politique comme genre de vie... Plus tard, j'ai pu être étonné de voir mes collègues géographes en situation de malaise vis-à-vis de la politique. Membre ou non d'un parti, agité ou contemplatif, plutôt satisfait ou plutôt mécontent des évolutions en cours, je me suis toujours senti à l'aise avec l'idée, qui est pourtant aussi un mythe, que j'étais actif, que j'étais, d'une manière ou d'une autre, un citoyen. Concrètement, outre un plaisir sans cesse renouvelé à lire les journaux, cela s'est traduit par un scepticisme envers les conceptions conspiratoires ou paranoïaques de la marche du monde, envers celles qui réduisaient la politique à des institutions omnipotentes, des personnages tireurs de ficelles, une mise en scène déjà écrite. Cela a signifié aussi pour moi, profondément, et malgré certaines maladresses de plume, qu'il ne fallait pas mélanger les genres. Lorsque je polémiquais au nom du marxisme avec d'autres géographes, je m'employais à distinguer les dispositions politiques des chercheurs, favorables ou non à la recherche, et la recherche elle-même ; j'étais surpris que, lorsque le problème dépassait les limites nationales, on s'en remît à la géographie, comme à un recours, à une médecine du social : je savais que le champ de la politique, c'était, par définition, la terre entière. J'étais irrité qu'on pût attribuer à la géographie une mission politique directe : je pensais que la politique et la géographie étaient deux astres, brillants tous deux, mais chacun sur sa propre orbite, chacun occupé à ses propres révolutions.

...Et le bel aujourd'hui.

11 Dès mes premières années, le présent était en somme, je l'ai dit, une esthétique dont chaque touche nouvelle accréditait l'idée d'un « art cinétique » improvisé. Mais il y a eu aussi l'esthétique comme présent. Cela a commencé dès les années soixante avec les films de Jean-Luc Godard vus en famille et âprement discutés ensuite. Puis la lente appropriation, en suivant le laborieux cheminement des programmes du lycée, de l'histoire de la musique et de la littérature, et en continuant sur la lancée vers ceux dont l'école ne nous parlait pas ou peu, jusqu'à ceux qui inventaient, maintenant: Iannis Xénakis ou Krzysztof Penderecki que je découvrai, un peu par hasard; Nathalie Sarraute ou Alain Robbe-Grillet, dont Les gommes étaient un classique familial, relayaient Jean-Paul Sartre, Albert Camus et Roger Vailland, déjà classiques mais encore contemporains. Le théâtre commença tôt et je pus encore voir Jean Vilar jouer du Brecht au TNP ; c'était peut-être là, à Chaillot ou dans les salles de banlieue, que je 
ressentais le plus l'innovation en action. Cela fut encore renforcé par la fréquentation de l'Ircam, des spectacles de danse contemporaine, des mises en scène iconoclastes d'opéra et des musées d'art moderne.

Tout cela semble on ne peut plus courant, banal ? En soi, peut-être, à ceux-ci près que beaucoup de nos contemporains adoptent une pratique patrimoniale de la consommation esthétique : n'est légitime que ce qui est "classique ", à l'exception de variétés de toute sorte. L'intéressant, en l'espèce, c'est la généralisation de l'idée d'« avant-garde » esthétique à toute création. J'ai vécu, je vis dans un univers mental où le degré d'innovation constitue un principe fondamental de séparation et, disons-le, de distinction des pratiques sociales. Si la répétition l'emporte sur l'invention, c'est pour moi une catastrophe artistique, mais aussi scientifique et peut-être même éthique. Créer, c'est une joie personnelle et une chance sociologique ; c'est aussi une sorte de contrainte, de contrat implicite signé avec les autres membres de la société. Celle-ci souffre d'être embourbée dans ses images anciennes qui la tirent en arrière et son mouvement en est freiné ; celui qui peut doit en proposer de nouvelles. Il ne s'agit pas bien sûr d'exalter le "nouveau à tout prix », c'est-à-dire les fausses nouveautés qui ne sont que clinquant badigeon sur l'ancien, modes cycliques, hommage du vice de la fantaisie à la vertu du classique. Mais il y a les vraies novations, celles qui ne nous flattent pas mais nous dérangent et changent notre regard, nous forcent à voir des réalités inédites et inouïes de nous-mêmes et du monde.

On comprend que, avec ces idées en tête, j'aie à chaque fois préféré la production sur la reproduction. Que j'aie été sévère, aussi, avec ceux qui se croyaient autorisés par leur âge, leur fonction ou d'autres intérêts, à se délier de l'obligation d'inventer.

Saint-Ouen, Fontenay-aux-Roses, Paris

Depuis ma naissance, j'ai habité sept logements, mais situés dans trois communes seulement: un à Saint-Ouen, dans l'actuelle Seine-Saint-Denis (1952-56); deux à Fontenay-aux-Roses dans les Hauts-de-Seine (1956-72 et 1972-74); quatre à Paris (1974-77, 1977-79, 1979-85, 1985-). Ces trois lieux appartenant à une même aire urbaine ne demandent qu'à raconter des histoires de stratégie et d'identité spatiales. Faut-il les écouter? Au moins en partie, avec l'idée de trouver dans le rapport à l'espace, ici à l'espace de l'habitat, une dimension du rapport à la société.

Le parcours peut se lire en écoutant le langage des acteurs: Saint-Ouen, lieu de la pauvreté, dans le logement et l'environnement urbain, assumé sans état d'âme par de jeunes adultes qui s'inséraient ainsi avec effort et plaisir dans la contre-société prolétarienne. Fontenay-aux-Roses, lieu fonctionnel, doté de confort et de proximités sociales favorables, mais dépourvu de rêves, ceux-ci s'installant d'emblée au centre. Paris, lieu nécessaire devenant espace ouvert, vrai début d'un itinéraire psychique et sociologique.

En ce sens, il s'agit bien d'une mobilité stratégique, mais d'une stratégie en partie implicite et animée par des acteurs différents. Le dépôt d'autobus, le cimetière, les Puces - et la « Zone » que l'on voit dans le film Le doulos - m'avaient plutôt laissé un bon souvenir, mais c'était une époque tranquille sans école, sans violence sociale, de petites vacances sans lendemain. Le passage de Saint-Ouen à Fontenay constitue un « adieu au prolétariat » en pratique, mais non en théorie. Arguant de leur condition modeste de locataires (de mes grands-parents) et de leurs bas revenus, mes parents cultiveront visà-vis du voisinage pavillonnaire de cadres supérieurs une différence pleine de sens politique : cette « modestie » économique offre un zeste d'« alliance objective » avec les 
vrais pauvres de la commune, de plus en plus rares au fil des ans. En fait, la théorie et la pratique sont plus complexes qu'il n'y paraît - très proches, avec d'autres mots, de la représentation des intellectuels chez Bourdieu, «fraction dominée de la classe dominante " investissant massivement son petit capital économique dans les biens culturels. L'importance des consommations culturelles, voyages compris, se retrouve dans la conception de l'habitat: on ne vantera pas, bien au contraire, la composition sociale de la population, mais on se félicitera de la présence de lycées, et de «bons » lycées, qui lui sont intimement liés.

17 Pourtant Fontenay-aux-Roses n'est qu'un sas. Ce n'est sans doute plus le «Capoue merdeux » dont parle Céline, mais cela reste une sous-ville sans génie, dont les terrains vagues et les cabanes magiques qu'on peut y construire constituent le principal attrait. Dans cette "banlieue Sud», les distances sont inutilement longues, les densités invariablement faibles, la « ligne de Sceaux », ce faux métro désespérément lent. Paris est l'Eden. Il ne sera pour un temps qu'accessible de l'extérieur, mais réussira à devenir le lieu de tous les plaisirs de la vie, qui sont plaisirs de la ville: métro, cinémas, restaurants, grands magasins, librairies. De l'avenue du Général-Leclerc, la centralité se déplace vers Luxembourg dont la gare, aux allures de catacombe, prend des allures de temple initiatique et le fracas des trains qui s'y jettent, de pompeux Alléluia. Paris fut cela, la terre promise point encore méritée. Un jour, on y vivrait.

Un jour, en 1968, j'y ai d'abord passé quelques nuits. Puis j'y suis allé tous les jours pour y faire mes études. Puis j'y ai vécu. C'était aussi bien que prévu, mais l'endroit était plus riche encore que l'on ne pouvait l'imaginer. Telle une construction à la Borges, il contenait aussi tout ce qui lui avait paru extérieur. Ce n'était pas qu'une vitrine, c'était un immense grand magasin aux rayons, beaux ou laids, toujours plus nombreux, toujours réaménagés. C'était un monde aussi grand que le monde et il fallait, écoutant Calvino, se contenter d'y vivre et d'en être content.

19 La grande ville était pour une part, c'est vrai, l'expression de réalités sociales qu'elle n'avait pas elle-même produites. Et il $\mathrm{y}$ avait plusieurs espèces d'urbains, certains auxquels on souhaitait s'identifier, d'autres non. Mais la grande ville coûtait cher et choisir d'y habiter avait un sens. Paris-terre promise, c'était aussi cela, probablement : conquérir une distinction proprement urbaine, qui était comme le couronnement de toutes les pratiques culturelles qui s'y déroulaient. Le capital social spécifique qu'apportait Paris, j'en vérifiai l'existence dans d'autres métropoles où j'eus la surprise de me retrouver chez moi, dès le premier pas. L'urbanité, ce n'était pas seulement un bien social négociable, c'étaient aussi des pratiques, des valeurs, une conception de la vie en société. Lorsque je compris que cette réalité était de nature spatiale, qu'une chose aussi essentielle pour ma place dans le monde faisait aussi partie de mon métier de géographe, quel événement, quelle divine surprise ! Ce fut un renouvellement de mon " inspiration » professionnelle, jusque-là surtout nourrie de paysages ferroviaires et de frontières d'État. Le déclic se fit peut-être en voyant Alice dans les villes de Wim Wenders ou Dans la ville blanche d'Alain Tanner: l'émotion esthétique y rejoignait l'aventure biographique et la construction cognitive. Lorsque, pour rédiger mon mémoire de maitrise sur le RER, je déambulais dans les couloirs accueillants ou intimes de la ville souterraine, je savais déjà, comme les héros de Subway, que là plus que partout ailleurs était la vraie vie.

Une égohistoire cognitive 
21 L'hypothèse avancée ici est qu'un changement de dominance est repérable dans ma vie intellectuelle à la fin des années soixante-dix. Ce changement était préparé par l'héritage familial et esquissé depuis que j'étais enfant. Il a été favorisé par un dispositif déjà présent, et qui n'a cessé de se renforcer, d'intégration d'éléments étrangers. Ce changement était donc déjà engagé au début de ma vie professionnelle.

Le dispositif familial se révèle décisif dans cette mutation. On a vu qu'une dimension essentielle de la vie de mes parents a consisté dans le passage d'un holisme (ethnicoreligieux) à un autre (étatico-monétaire), accompagné d'un desserrement de toute communauté. En outre, la dimension économique de l'identification (la «lutte des classes ») est valorisée dans le discours politique mais niée dans la pratique par une rupture essentielle de mes parents avec leurs parents, qui eux-mêmes vivaient la contradiction sans la trancher: mes parents choisissent la culture contre la «boutique » et disqualifient tout projet d'ascension sociale par accumulation de capital économique. Ils tentent plus ou moins de se faire croire qu'ils sont «modestes ", c'està-dire pauvres comme des ouvriers, ce qui a été vrai à un moment de leur vie. Mais le primat qu'ils accordent à la culture et aux valeurs "post-matérialistes" est sans ambiguïté, beaucoup moins équivoque ou polysémique que l'attitude d'une aristocratie intellectuelle "dynastique ", tôt ou tard impliquée dans une stratégie de constitution et de reproduction d'un patrimoine, y compris économique. La " pauvreté » choisie (mon père a quitté une carrière universitaire pour devenir "permanent» politique) et assumée sans douleur engendre un type-idéal particulièrement épuré. La place est donc dégagée pour l'adoption d'une dominance "systémique", qui met en avant les productions non réductibles à une chaîne linéaire ("objectale») et réalisées par l'ensemble de la société : individu, ville, culture, la société elle-même dans sa densité et sa diversité, dans son historicité.

La conjonction de l'école républicaine, de la Résistance et de la composante émancipatrice du discours marxiste permettent la montée en puissance d'universalismes qui sont appelés, par leur propre logique, vers des conceptions et des pratiques de la liberté individuelle. Cette évolution sera freinée par la nature holistique de l'allégeance politique au communisme, mais, sans tomber dans une tautologie rétrospective, on peut penser qu'il était on ne peut plus probable que cette barrière saute, un jour ou l'autre. A cela s'ajoute une culture (franco-judéo-méditerranéenne ?) de la politique, de la discussion et de la mondialité que j'ai résumée par le terme cosmopolis. On a donc tous les ingrédients pour une orientation dynamique, relativement dégagée de frottements liés à des ancrages sociaux qui auraient porté vers la reproduction. Mes parents ont assumé jusqu'au bout leur choix, exprimé dans la nation républicaine-universaliste et incarné dans le communisme. Il ont pu ainsi liquider leur déracinement. Je me retrouvais, à quelques scories près, libre de me désenraciner.

Dans sa dimension individuelle, la nouvelle dominance tire ses origines de deux sources contradictoires : une "théorie du Sujet » fondée sur le libre-arbitre et une approche holistique de la société liée à une vision téléologique de l'histoire. La « synthèse » sera en fait un dépassement de ces deux matériaux antinomiques. Pour produire cet « individualisme sociétal », il aura fallu mobiliser un troisième élément, l'expérience, plus précisément la lecture d'une expérience de l'action sociétale de l'individu. Cette expérience provient pour une part du domaine cognitif-objectif (apprentissages scolaires, débats familiaux, lectures, voyages, connaissance de la vie politique), mais 
aussi d'autres secteurs de la personnalité : des relations intersubjectives (pratique, au nom de la défense de l'intégrité individuelle, d'une liberté face aux communautés), de l'éthique (tentative de dépassement, à partir du rejet de la notion de péché, de la contradiction entre la morale et l'intérêt) et de l'esthétique (primat de l'innovation sur la reproduction). Les évolutions dans les différents secteurs se sont peu à peu rencontrées grâce à des désenclavements, détaillés plus loin, sans lesquels une lecture unifiée de la transformation eût été impossible. Or un tel discours sur les changements que l'on subit («conscience des transformations du moi ») est à son tour moteur de nouvelles transformations, par diffusion du nouveau modèle dans l'ensemble de l'activité psychique.

La géographie actuelle me semble souffrir d'une mobilisation insuffisante des connaissances qu'ont - ou que pourraient acquérir - les géographes dans les différentes sciences sociales. Beaucoup des travaux les plus brillants dans notre discipline me semblent en partie diminués, et déqualifiés sur le marché, du fait que leurs auteurs ne se sont jamais vraiment dit que leur culture, dans le dessein de faire une bonne géographie, devait aller au-delà de la seule géographie. On me répondra " area studies " ou " études régionales ». Je crains que ce ne soit le dernier refuge de l'enclavement. Le développement en rhizome, c'est-à-dire par expansion floue des connaissances, sans redéfinition des cadres de pensée et donc sans rupture, va faire que le spécialiste d'un lieu saura "tout» sur ce lieu, y compris au moyen d'«informations» provenant d'autres disciplines. Mais va-t-il pour autant saisir l'occasion pour accepter la subversion qu'apportent sur son « terrain » d'autres concepts, d'autres conceptions?

Au total, accorder la prééminence (et non l'exclusivité, qui serait aberrante du point de vue de la connaissance) au network, à la reformulation permanente des structures de la pensée, à la "tectonique casssante" plutôt qu'au rhizome permet une meilleure falsifiabilité 1 . Les messages informant de l'existence d'impasses théoriques parviennent rapidement au centre et le mettent en cause même s'ils sont originaires d'une lointaine périphérie. Pour des raisons qui tiennent à ma formation et à mon expérience, j'ai d'emblée privilégié cette disposition. Dans un premier temps, cela a renforcé une attitude dogmatique résiduelle de la première dominance: c'était ce qu'on appelait autrefois l'« esprit de système ", le network clos : ce qui n'y entrait pas était rejeté sans discussion. Au contraire, la nouvelle dominance a pu se mettre en place puis fonctionner grâce à l'intégration des rhizomes dans une pratique du network ouvert: toute "racine adventice " était désormais considérée comme un signe possible d'inadéquation du système global et, après évaluation, soit elle était rejetée comme non pertinente, soit elle était intégrée et «naturalisée », soit enfin elle devenait le poison létal du système entier et imposait une recomposition générale.

C'est ce qui s'est passé lorsque j'ai compris, sous des influences diverses, internes et externes à la pratique scientifique, que l'action individuelle jouait un rôle essentiel dans le fonctionnement et le développement de l'espace urbain. De proche en proche, ont été atteints par ce "virus "la définition de la ville, les notions d'acteur spatial et de stratégie spatiale, la relation entre espace civil et espace politique dans la ville, l'idée de démocratie urbaine. La place nouvelle que je découvrais à l'individu et qui se révélait elle-même en pleine mutation me contraignait aussi à modifier l'organisation des raisonnements et l'agencement des causalités dans l'ensemble de ma démarche géographique. La lecture des changements de la carte électorale se trouvait 
profondément affectée si l'on faisait intervenir des processus de sortie de la logique communautaire. On pourrait rétablir le fil des événements de manière comparable à propos de l'échelle mondiale : le déclic, dans ce cas, ce fut la notion de société. Voilà une échelle qui semblait résister à un concept dont pourtant mes recherches me conduisaient à renforcer sans cesse la sphère d'application. Je suis donc parti de ce manque, de ce vide : le monde était une échelle où la société, ça ne marchait pas. Je fus alors contraint de remettre en question un édifice, ou plutôt ce qui était alors une construction de fortune, ou plus précisément encore ce qui ressemblait à une bouillie pâteuse, un apple crumble où l'on rencontrait dans un ordre approximatif, à la place des morceaux de pommes, ici des États, là l'économie, ailleurs des «cultures» et encore ailleurs la «culture». J'aurais pu vivre longtemps avec ce crumble, pâtisserie au demeurant délicieuse. Il se trouve que, en pareille circonstance, je ne peux pas supporter longtemps la vision d'un tel chaos. La tectonique cassante, c'est cette force maligne, ce méchant démon qui tire sur la pièce dont dépendait l'équilibre fragile d'un échafaudage parfois laborieusement dressé et qui, devant la scène de désolation d'une théorie brisée en mille morceaux, s'écrie en ricanant : «Et maintenant, qu'est-ce que tu proposes?» Pour échapper à un nouveau ricanement du démon, je me sens alors obligé de proposer quelque chose.

Si l'intégration du network dans la nouvelle dominance était parfaitement réalisée, la " machine à se changer " y occuperait la place centrale. Le risque affectif permanent, le "dérangement» esthétique maximal, une éthique associant implacablement la responsabilité individuelle au devenir collectif seraient les « correspondants » logiques de la tectonique cassante repérée dans le travail théorique. Pour vivre ainsi, il faut être persuadé que l'on peut se permettre de "mourir", de voir ce qu'on croyait, aimait, cultivait, pensait, disparaitre avec celui que l'on était dans les profondeurs - pour "renaître », réinvesti de ce qui a disparu, et d'un petit quelque chose d'autre. C'est imaginable, c'est même réalisable, mais c'est extrêmement fatiguant.

(...)

31 Je suis probablement moins éloigné de beaucoup de mes contemporains que je ne le crois...

Ma seule singularité est sans doute que les discours que je me suis construits pour me penser stable visent à être à la fois cohérents et globaux et que, malgré cette rigidité apparente ou peut-être à cause d'une succession de rigidités, leur capacité d'intégration sort plutôt renforcée des informations nouvelles qui leurs parviennent. J'ai donc la sensation que les circonstances contingentes qui d'une part ont tendu à me dégager d'appartenances diverses, d'autre part m'ont fourni des instruments utiles pour penser le réel, me donnent l'occasion d'exprimer, avec une certaine netteté, une configuration émergente, commune à beaucoup, mais sous une forme moins visible ou moins explicitée. J'ai à peu près cessé, je crois, de manifester l'arrogance un peu théâtrale qui fut sans doute une manière de paraître plus assuré ; pourtant cette posture saillante que je revendique aujourd'hui sérieusement, pour le fond de ce que j'ai à dire, peut continuer d'irriter, j'en conviens.

(...)

Ce que chercher veut dire 
... Chaque fois que l'on termine un travail, il faudrait, au risque de miner l'inévitable sentiment de légèreté que l'on éprouve alors, se poser la question sans détour : «Qu'en penseras-tu dans vingt ans?»

Ce que j'en pense vingt ans plus tard, c'est finalement ceci.

Pour celui que j'étais à ce moment-là, la géographie française de 1972 offrait un objet finalement trop tentant, un vieux jouet à casser, une maison neuve à construire. Ces deux plaisirs se sont, je le soupçonne, un temps mêlés, inconsciemment, dans mon esprit.

(...)

Non plus comme chercheur mais comme « citoyen ", j'ai progressivement participé à la reconstruction d'un édifice de pensée et d'action qui s'en prend non seulement à la « grille » marxiste, mais aussi à ce qu'a été la gauche depuis les débuts du mouvement ouvrier en Europe. Ce n'est donc pas d'une attitude en demi-teinte qu'il s'agit. Mais on comprend bien ces temps-ci que la radicalité d'une démarche critique se mesure aussi à la capacité de faire le tri, et notamment à l'aptitude à replacer les productions intellectuelles qu'on critique dans l'histoire de la pensée, même et surtout si celles-ci prétendaient à une validité détachée de l'histoire. Dans cet esprit, contrairement à ces géographes anglo-saxons qui, après avoir embrassé successivement la «révolution quantitative " et le "primat de la lutte des classes ", cherchaient une étiquette de nouveau à la mode, je n'ai pas été tenté par le prurit «post-moderne » des années quatre-vingt.

9 C'est que, après avoir filtré et réorganisé le stock de représentations qui m'était accessible, je me suis choisi une ascendance fondatrice : la pensée gréco-chrétienne et les Lumières, les courants épistémologiques de la philosophie occidentale, les sciences sociales contemporaines, et en leur sein retrouvé la géographie d'aujourd'hui constituent pour moi un socle sur lequel bâtir de nouvelles constructions cognitives et non un obstacle à écarter. L'historicité intégrale de l'homme et de sa raison me semblent une matrice efficace, jusqu'à présent, pour répondre aux défis cognitifs de nos sociétés. Elle est capable, en s'enrichissant, d'animer de nouvelles "pancraces épistémologiques", ouvrant sur des "explorations axiomatiques" innovantes et porteuses de "réseaux théoriques $»^{2}$ inédits. Ce mouvement incessant d'arrachement au foisonnant et à l'informe est aussi un parcours éthique, esthétique, intime.

Tant que je conserverai l'illusion heureuse qu'il suffit de participer à cette aventure, fût-ce sans toujours savoir où l'on va ni comment l'on y va, pour se changer beaucoup soi-même tout en changeant un peu l'état des connaissances, j'espérerai en être, pour un moment encore.

\section{NOTES}

1. Ce mot est employé au sens où l'utilise Popper, c'est-à-dire pour désigner la possibilité, pour un énoncé scientifique, d'être réfuté. 
2. Ces trois vocables reprennent les titres des subdivisions de la deuxième partie de L'espace légitime (NdE).

\section{RÉSUMÉS}

La subjectivité des chercheurs fait partie des facteurs de production de la recherche. Pour comprendre comment fonctionne cette « subjectivité cognitive ", l'auteur s'est efforcé de cerner les moments et les attitudes qui ont compté le plus dans sa vie intellectuelle. Ce sont quelques extraits de cette « ego-géographie »qui sont présentés ici.

Self-portrait with geography.

Researcher's subjectivity is part of the factors of production of research. To understand how that " cognitive subjectivity » operates, the author has tried to delimit the moments and attitudes which have most counted in his intellectual life. Some extracts of that " ego-geography " are here presented.

INDEX

Mots-clés : autobiographie cognitive, Subjectivité, Itinéraire, Epistémologie

Keywords : cognitive autobiography, Subjectivity, Itinerary, Epistemology

\section{AUTEUR}

\section{JACQUES LÉVY}

Chargé de recherches au CNRS (STRATES) et professeur à l'Institut d'études politiques de Paris, il travaille sur la dimension du politique appliquée à des objets aussi divers que la ville, l'Europe, la mondialité. Il anime, depuis son lancement, la revue EspacesTemps. 